Draft Version July 31, 2021

Preprint typeset using $\mathrm{IAT}_{\mathrm{E}} \mathrm{X}$ style emulateapj v. 5/2/11

\title{
CONSTRAINING THE FREQUENCY OF FREE-FLOATING PLANETS FROM A SYNTHESIS OF MICROLENSING, RADIAL VELOCITY, AND DIRECT IMAGING SURVEY RESULTS
}

\author{
Christian Clanton ${ }^{1,2,3}$, B. Scott Gaudi ${ }^{3}$ \\ Draft version July 31, 2021
}

\begin{abstract}
A microlensing survey by Sumi et al. (2011) exhibits an overabundance of short-timescale events $\left(t_{E} \lesssim 2\right.$ days) relative to that expected from known stellar populations and a smooth power-law extrapolation down to the brown dwarf regime. This excess has been interpreted as a population of approximately Jupiter-mass objects that outnumber main-sequence stars by nearly twofold; however the microlensing data alone cannot distinguish between events due to wide-separation $(a \gtrsim 10 \mathrm{AU})$ and free-floating planets. Assuming these short-timescale events are indeed due to planetary-mass objects, we aim to constrain the fraction of these events that can be explained by bound but wideseparation planets. We fit the observed timescale distribution with a lens mass function comprised of brown dwarfs, main-sequence stars, white dwarfs, neutron stars, and black holes, finding and thus corroborating the initial identification of an excess of short-timescale events. Including a population of bound planets with distributions of masses and separations that are consistent with the results from representative microlensing, radial velocity, and direct imaging surveys, we then determine what fraction of these bound planets are expected not to show signatures of the primary lens (host) star in their microlensing light curves, and thus what fraction of the short-timescale event excess can be explained by bound planets alone. We find that, given our model for the distribution of planet parameters, bound planets alone cannot explain the entire excess without violating the constraints from the surveys we consider, and thus some fraction of these events must be due to free-floating planets, if our model for bound planets holds. We estimate a median fraction of short-timescale events due to free-floating planets to be $f=0.67(0.23-0.85$ at $95 \%$ confidence $)$ when assuming "hot-start" planet evolutionary models and $f=0.58$ (0.14-0.83 at $95 \%$ confidence) for "cold-start" models. Assuming a delta-function distribution of free-floating planets of mass $m_{p}=2 M_{\text {Jup }}$ yields a number of free-floating planets per main sequence star of $N=1.4(0.48-1.8$ at $95 \%$ confidence) in the "hot-start" case and $N=1.2(0.29-1.8$ at $95 \%$ confidence) in the "cold-start" case.

Subject headings: methods: statistical - planets and satellites: general - gravitational lensing: micro techniques: radial velocities - techniques: high angular resolution - stars: low-mass
\end{abstract}

\section{INTRODUCTION}

Deep optical and near-infrared photometric surveys to characterize the low-mass end of the substellar initial mass function (IMF) have identified populations of isolated, planetary-mass candidates in several nearby, young star-forming regions and clusters (Comeron et al. 1993; Nordh et al. 1996; Itoh et al. 1996; Tamura et al. 1998; Lucas \& Roche 2000; Zapatero Osorio et al. 2000, 2002; McGovern et al. 2004; Kirkpatrick et al. 2006; Luhman et al. 2006; Bihain et al. 2009; Burgess et al. 2009; Scholz et al. 2009, 2012a,b; Weights et al. 2009; Marsh et al. 2010; Quanz et al. 2010; Mužić et al. 2011, 2012, 2014, 2015). While a number of these photometrically-identified candidates have been met with some controversy in the literature concerning their youth (and thus low masses) or cluster membership (see e.g. Hillenbrand \& Carpenter 2000; Allers et al. 2007; Luhman et al. 2007a), recent studies, in particular those by the Substellar Objects in Nearby Young Clusters (SONYC) group, have focused on obtaining confirma-

2 NASA Ames Research Center, Space Science \& Astrobiology Division, Moffett Field, CA 94035, USA

3 Department of Astronomy, The Ohio State University, 140 W. 18th Ave., Columbus, OH 43210, USA
}

tion spectra of such candidates and have verified several free-floating, planetary-mass objects with masses as low as a few Jupiter masses (Scholz et al. 2009, 2012a,b; Mužić et al. 2011, 2012, 2015). Sumi et al. (2011) also present evidence for a large population of Jupiter-mass objects that are either wide-separation $(a \gtrsim 10 \mathrm{AU})$ or free-floating planets inferred from an excess of short events in the observed timescale distribution of a sample of microlensing events collected by the second phase of the Microlensing Observations in Astrophysics group (MOA-II; Sumi et al. 2003; Sako et al. 2008). Wyrzykowski et al. (2015) report that data from the third phase of the Optical Gravitational Lensing Experiment (OGLE-III; Udalski 2003) show a flattening in the slope of the observed event timescale distribution towards shorter timescales that is suggestive of a population of lenses similar to that reported by Sumi et al. (2011), although this flattening is only marginally significant due to uncertainties resulting from small-number statistics and a low detection efficiency to such shorttimescale events.

Comparing the occurrence rates of free-floating planets inferred by imaging surveys with those from microlensing is difficult, as the imaging surveys have sensitivities that cut off around 1-3 $M_{\text {Jup }}$ (and depend on the exact evolutionary model adopted), while Sumi et al. (2011) found 
that these objects (regardless of their boundedness) most likely have masses near (and probably below) the sensitivity limit of the imaging surveys at $1.2_{-0.7}^{+1.2} M_{\mathrm{Jup}}$. Nevertheless, in the SONYC survey of the young cluster NGC 1333, Scholz et al. (2012b) find that the occurrence rate of (photometrically-identified, spectroscopically confirmed) free-floating, planetary-mass objects relative to main-sequence stars is smaller than that inferred by the Sumi et al. (2011) microlensing study by a very large factor of some 20-50. Scholz et al. (2012b) argue that the star formation process extends into the planetary-mass regime, down to the planetary masses they are able to probe, and thus this large difference in inferred occurrence rates of free-floating planets must be due to a very large upturn in the mass function of compact objects below $\sim 3 M_{\text {Jup }}$ that is perhaps indicative of a different formation channel (assuming that microlensing and direct imaging surveys are probing an analogous population of compact objects). Alternatively, one might argue that young open clusters may have a different mass function than the objects in the Galactic disk and bulge that give rise to microlensing events.

On the other hand, the photometric survey of $\rho$ Oph by Marsh et al. (2010) find a much larger number of isolated, planetary-mass objects per main-sequence star. After integrating their inferred mass function (shown in their Figure 8) in the planetary regime, the lowest two bins between $7 \times 10^{-4} \lesssim M / M_{\odot} \lesssim 6 \times 10^{-3}$ (corresponding to roughly $0.7 \lesssim M / M_{\mathrm{Jup}} \lesssim 6$ ), and in the stellar regime, the highest three bins between $0.08 \lesssim M / M_{\odot} \lesssim 1.0$, we divide these values to estimate the implied number of free-floating planets per main-sequence star of $\sim 30$. This number is over an order of magnitude larger than that of Sumi et al. (2011) and larger than the results of Scholz et al. (2012b) by an even greater factor. This seems to suggest that either the formation of free-floating planets is extremely sensitive to the local environment, the Marsh et al. (2010) sample is contaminated (with background stars or due to mis-estimates of the ages and/or masses of the candidate free-floating planets; see e.g. Luhman et al. 2007a and Allers et al. 2007) since they lack spectroscopic validation for many of their candidates, or some combination thereof.

Broadly, there are two formation channels for freefloating planets, but there are issues with the theory and observations behind each. The first, as Scholz et al. (2012b) claim, is that these objects form as an extension of the star formation process, however the lower mass fragmentation limit predicted by models of collapsing clouds is uncertain (e.g. Silk 1977; Padoan et al. 1997; Adams \& Fatuzzo 1996) and may, in fact, be dependent on environment (e.g. Bate \& Bonnell 2005; also see Luhman et al. 2007b and Bastian et al. 2010 for a discussion of the substellar IMF and its universality). Secondly, if free-floating planets initially form from material in circumstellar disks (either by disk fragmentation or core accretion), they must be subsequently ejected out of the system via dynamical processes such as planetplanet scattering, mass loss during post-main-sequence evolution, or ionization by interloping stars.

The ejection of a $\sim$ Jupiter-mass planet via planetplanet scattering requires a close encounter with an- other planet with a mass at least a Jupiter mass or above, as the least massive body in such an encounter is nearly always the one ejected (see e.g. Ford et al. 2003; Ravmond et al. 2008). Thus, if planetplanet scattering were the dominant channel for formation of the population of (presumably) free-floating, Jupiter-mass planets inferred by Sumi et al. (2011), the frequency of Jupiter- and super-Jupiter-mass planets around low-mass stars must necessarily be high ( 50\%; Veras \& Raymond 2012), which is in significant disagreement with the predictions of core accretion theory (Laughlin et al. 2004) as well as observational results from microlensing (Gould et al. 2010; Cassan et al. 2012; Clanton \& Gaudi 2014b, 2016), radial velocity (Bonfils et al. 2013; Montet et al. 2014), and direct imaging (Lafrenière et al. 2007; Bowler et al. 2015) surveys. Additionally, ejection due to mass loss during post-mainsequence evolution only works for planets with very wide orbital separations ( $\sim$ several hundred AU) and requires (initial) host masses $\gtrsim 2 M_{\odot}$, and thus is not expected to produce free-floating planets at the required rate (Veras et al. 2011; Mustill et al. 2014).

Similarly, ionization by interloping stars requires initially wide planetary orbits and a dense stellar environment since the ionization time scales as $t_{\text {ion }} \propto \nu^{-1} a^{-2}$, where $\nu$ is the local stellar number density and $a$ is the semimajor axis (see Antognini \& Thompson 2016, and references therein). Antognini \& Thompson (2016) demonstrate that even in the case of the most optimistic interaction cross sections, $t_{\text {ion }} \sim 2 \mathrm{Gyr}$, implying that $\sim 10 \%$ of systems with planets on wide orbits would have been ionized in a cluster with an age of $200 \mathrm{Myr}$. In the field, these authors find $t_{\text {ion }} \sim 4 \times 10^{12} \mathrm{yr}$ and therefore $\lesssim 1 \%$ of wide-separation planetary systems would have been ionized in the lifetime of the Galaxy. Given current measurements of upper limits on the frequency of Jupiter- and super-Jupiter-mass planets with $a \gtrsim 10 \mathrm{AU}$ from direct imaging surveys of young FGK stars of $\lesssim 20-30 \%$ (Lafrenière et al. 2007; Biller et al. 2013) and young $M$ stars of $\lesssim 16 \%$ (Bowler et al. 2015), it does not seem likely that ionization (even in clusters) is able to produce the large numbers of free-floating planets inferred by Sumi et al. (2011), although (to the best of our knowledge) a robust, quantitative analysis has yet to be performed.

Thus, while it may be possible to explain the formation of the smaller population of free-floating, planetarymass objects observed by the SONYC group, the origin of the much larger population inferred by the Sumi et al. (2011) study remains elusive. One possible (and simple) solution could be that a majority of the planetary-mass objects needed to reproduce the over-abundance of shorttimescale microlensing events seen in the MOA-II data (Sumi et al. 2011) are not actually free-floating, but are gravitationally bound to host stars at wide enough orbital separations $(a \gtrsim 10 \mathrm{AU})$ that we do not expect to see signatures of the primaries (i.e. host stars) in a majority of their microlensing light curves and we do not expect them to be detected by direct imaging surveys (due to either lying outside the outer-working angles of such surveys, and/or having masses less than $\sim$ few Jupiter masses, below their detection limits).

In this study, we attempt to fit the observed timescale distribution with a standard lens mass function (here- 
after LMF) comprised of brown dwarfs, main-sequence stars, white dwarfs, neutron stars, and black holes, along with a population of wide-separation, bound planets that is known to be consistent with the results of microlensing, radial velocity, and direct imaging surveys. In Clanton \& Gaudi (2016), we demonstrated that there is a single planet population, modeled by a simple, joint power-law distribution function in planet mass and semimajor axis, that is simultaneously consistent with several representative surveys employing these three distinct detection techniques. Some fraction of such a planet population would produce detectable, short-timescale microlensing events that are well-fit by a single lens model, similar in nature to the 10 observed events with $t_{E}<2$ days in the MOA-II data that Sumi et al. (2011) present. We determine the expected timescale distribution for the combination of our adopted LMF and our planet population model and compare with the observed distribution to estimate the fraction of short-timescale events that are due to free-floating planets.

The remainder of the paper is organized as follows. We detail the properties of the Sumi et al. (2011) microlensing event sample and review their analysis to infer the existence of an abundant population of either wideseparation or free-floating planets in Section 2 . We describe the different channels for distinguishing microlensing events due to free-floating planets from those due to bound planets in Section 3. We detail the methodologies we employ in this study in Section 4 and present our results, together with discussion, in Section 5. Finally, we provide a summary of this work in Section 6 .

\section{THE ABUNDANCE OF WIDE-SEPARATION OR FREE-FLOATING PLANETS INFERRED BY MICROLENSING}

Sumi et al. (2011) select a sample of 474 wellcharacterized microlensing events from the 2006-2007 MOA-II data set. Here, well-characterized means that each light curve was determined to contain a genuine microlensing event that is distinguishable from intrinsically variable stars and other artifacts (e.g. cosmic rays, background supernovae). Sumi et al. (2011) require that each light curve have a single brightening episode consisting of more than three consecutive measurements (that are each $>3 \sigma$ above a constant baseline) and be "well-fit" by a theoretical microlensing model with a well-constrained (fractional error $\leq 0.5$ ) Einstein crossing time, $t_{E}$ (see Sections 2 and 3 and Table 2 of the Supplemental Materials of Sumi et al. 2011 for a detailed description of their selection criteria and their particular definition of "well-fit").

Of these 474 microlensing events, 10 of them have timescales between $0.3 \leq t_{E} /$ days $\leq 2$. For a lens mass $M_{\mathrm{L}}$, lens-source relative parallax $\pi_{\text {rel }}$, and lenssource relative proper motion $\mu_{\text {rel }}$, the Einstein crossing time scales as $t_{E} \propto\left(M_{\mathrm{L}} \pi_{\text {rel }}\right)^{1 / 2} / \mu_{\text {rel }}$, which means that for typical values of $\pi_{\text {rel }}$ and $\mu_{\text {re }}$, microlensing events with timescales $t_{E} \lesssim 2$ days would indicate planetarymass lenses. Indeed, Sumi et al. (2011) fit the observed

${ }^{4}$ For a standard Galactic model (e.g. Han \& Gould 1995a b, 2003), $\pi_{\text {rel }}$ is expected to vary from 0.043 mas to 0.21 mas and $\mu_{\text {rel }}$

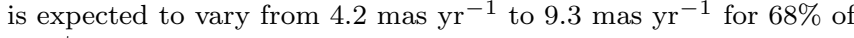
events. timescale distribution with an ensemble of simulated microlensing events appropriately weighted by their event rate as well as their detection efficiency as a function of $t_{E}$ (constructed by adopting a model of the Galaxy and a LMF over a mass range of $0.01 \leq M_{\mathrm{L}} / M_{\odot} \leq 100$ ), and found an expected number of events with timescales $t_{E}<2$ days due to stellar, stellar remnant, and brown dwarf lenses to be either 1.5 or 2.5 (depending on their specific choice of form for the LMF). In either case, there is a clear overabundance of short-timescale microlensing events that is unexplained by such a model.

Sumi et al. (2011) found that the fit to the overall timescale distribution is significantly improved when they included a population of planetary-mass objects as an extension to their canonical LMF (see their Figure 2). Sumi et al. (2011) assumed that the population of planetary mass objects has a $\delta$-function mass distribution and found the value that most closely reproduces the observed timescale distribution to be $m_{p}=1.1_{-0.6}^{+1.2} M_{\text {Jup }}$. They also infer that the relative number of such objects to main-sequence stars $\left(0.08 \leq M_{\star} / M_{\odot} \leq 1.0\right)$ is $1.9_{-0.8}^{+1.3}$ or $1.8_{-0.8}^{+1.7}$, again depending on the specific form of the mass function for the higher-mass lenses $\left(M_{\mathrm{L}} \geq 0.08 M_{\odot}\right)$. Sumi et al. (2011) also tested a powerlaw mass function for the population of planetary-mass objects of the form $d N_{\mathrm{pl}} / d \log m_{p}=m_{p}^{1-\alpha_{\mathrm{pl}}}$ over the mass range $10^{-5} \leq m_{p} / M_{\odot} \leq 0.01$ (corresponding to $3 M_{\oplus} \lesssim m_{p} \lesssim 11 M_{\mathrm{Jup}}$ ) and found the slope that most closely reproduces the observed timescale distribution to be $\alpha_{\mathrm{pl}}=1.3_{-0.4}^{+0.3}$, from which they infer the relative number of planetary mass objects to main-sequence stars to be $5.5_{-4.3}^{+18.1}$. Sumi et al. (2011) note that while this power-law model has a maximum likelihood value that is $75 \%$ smaller than that of their $\delta$-function planet mass model, it also has one fewer free parameter and is thus (formally) a slightly better fit. In the case of the $\delta$-function model, there are two additional free parameters, the mass and normalization, whereas in the case of the power-law model, the only additional free parameter is the slope (the normalization is included in the overall normalization of their LMF).

Although it is clear that a majority of the 10 events with $t_{E}<2$ days must be due to planetary-mass lenses (if they are indeed due to microlensing and standard models for the distributions of $\pi_{\text {rel }}$ and $\mu_{\text {rel }}$ are accurate), it is not certain whether these objects are gravitationally bound to a host star or if they are free-floating planets. Sumi et al. (2011) searched for signatures indicative of the presence of a host star in the light curves of the short-timescale events and found nothing (see Section 3 for details on how to distinguish wide-separation planets from unbound planets), but were able to place limits on the projected separation (in units of the Einstein radius), $s$, of each planet from the host lens under the assumption that one exists (see their Table 1). These limits range between $2.4 \leq s_{\min } \leq 15.0$, which roughly corresponds to semimajor axes between $6.7 \lesssim a_{\min } / \mathrm{AU} \lesssim 42$ assuming a typical primary lens, event parameters, and the median projection angle of a circular orbit, with a median value of $s_{\min } \simeq 4.2\left(a_{\min } \simeq 12 \mathrm{AU}\right)$. Here, the variables $s_{\min }$ and $a_{\min }$ represent the minimum values of the projected separation and corresponding semimajor axis (assuming a randomly-oriented, circular orbit), respec- 
tively, that would be plausible given the non-detection (at the $2 \sigma$ level) of features in the microlensing light curves that would indicate the presence of a host star. We note that Sumi et al. (2011) did find three shorttimescale events that clearly showed both binary lens caustic crossing features and very low-amplitude signals due to lensing by the primaries (see Bennett et al. 2012 for an analysis of these three events, one of which was the first planetary microlensing event in which the host star was detected only through binary lensing effects, MOAbin-1), but none of these passed all their selection criteria and made it into their final sample.

Since these microlensing data alone are insufficient to constrain the fraction of the population of planetarymass lenses that are truly unbound, Sumi et al. (2011) consulted results from the Gemini Deep Planet Survey (GDPS; Lafrenière et al. 2007) that place upper limits on the frequency of wide-separation $(10 \lesssim a / \mathrm{AU} \lesssim 500)$ Jupiter- and super-Jupiter-mass planets. Using the information contained in Figure 10 of Lafrenière et al. (2007), Sumi et al. (2011) estimated that $<40 \%$ of the population of planetary-mass objects required to explain the overabundance of short-timescale microlensing events can be gravitationally bound to a host star at separations between $10-500 \mathrm{AU}$, assuming any such planets have a uniform distribution of $\log a$.

However, we argue that the use of the full GDPS sample to constrain this fraction of bound planets is not correct (although we show in Section [5 that our final result is actually consistent with the fraction estimated by Sumi et al. 2011). The stellar samples of the Sumi et al. (2011) survey and the GDPS are quite different, and thus the upper limits on planet frequency derived from the full GDPS sample are not necessarily representative of those for only the M stars. This is an important point because microlensing samples are dominated by low-mass lens stars due to the fact that the rate of microlensing events depends explicitly on the mass function of lenses, which is weighted in favor of low-mass stars. On the other hand, the GPDS sample is comprised primarily of FGK stars, with a smaller number of M stars; of the full sample of 85 stars, just 16 are classified with M spectral types. While these stars are generally young, they are old enough that the lower-mass stars probably have spectral types that are not significantly different than that they will have when they fall on the main sequence. By comparing their observed $K$-band magnitudes to that predicted by stellar isochrones of M stars at similar ages, we argue that most, if not all, of the stars classified with $M$ spectral types in the GDPS sample have a high likelihood of being an analogous population to the low-mass stars that produce the majority of microlensing events toward the Galactic bulge (see Clanton \& Gaudi 2016 for discussion). This issue has been pointed out in Quanz et al. (2012), who perform a more careful analysis using the GDPS constraints for just the $\mathrm{M}$ stars to estimate the upper limit on the fraction of the population of planetary-mass objects responsible for the observed short-timescale events that are bound to a host star, $f_{\max }$. These authors found a value of $f_{\max }=0.78$ (at $95 \%$ confidence) if these planets have a typical mass of $1 M_{\mathrm{Jup}}$ and have separations equal to $a_{\mathrm{min}}$ that Sumi et al. (2011) calculate for each of the shorttimescale events. If the planets are located at separations of $2 a_{\min }$, then $f_{\max }=0.49$. Of course, the true planet population (assuming such a bound population exists) will have some distribution of separations, and will directly affect the value of $f_{\max }$. Another potential issue affecting both the Sumi et al. (2011) and Quanz et al. (2012) analyses is that the GDPS sensitivites (in terms of planet mass) they employ assume "hot-start" planet evolutionary models (Baraffe et al. 2003), which represent the most optimistic predictions for detecting planetary companions via direct imaging.

In this paper, we perform a thorough joint analysis of microlensing, radial velocity, and direct imaging constraints, selecting samples of stars similar to that probed by the Sumi et al. (2011) survey and considering both "hot-" and "cold-start" planet evolutionary models to determine the expected timescale distribution of wide-separation, bound planets whose microlensing light curves reveal no evidence of the host stars they orbit. We will also do a more robust analysis than those of either Sumi et al. (2011) or Quanz et al. (2012) by including a distribution of planetary separations, including an outer cutoff semimajor axis for the population, to compute the fraction of the short-timescale events that are due to freefloating planets.

\section{DISTINGUISHING BETWEEN MICROLENSING EVENTS DUE TO WIDE-SEPARATION AND FREE-FLOATING} PLANETS

In a microlensing event due to a wide-separation $(s \gg$ 1 , where $s$ is the projected separation in units of the Einstein radius) planet, evidence for boundedness can be obtained through three channels: 1) observation of a relatively long-timescale (and likely low-magnification) bump due to the source trajectory passing near enough to the primary to produce a detectable magnification, 2) observation of anomalies near the peak of the light curve due to the source passing near (or crossing) the planetary caustic (Han \& Kang 2003), and/or 3) detecting blended light from the primary. The latter channel requires data of sufficient angular resolution to resolve out any unrelated stars, so that any additional flux above that of the source is due to the host lens (or a companion to the lens or source). MOA-II data typically have seeing ranging between $1.9-3.5$ arcsec, with a median of $\sim 2.5$ arcsec (Bond et al. 2001; Sumi et al. 2003), and thus any detected blend flux could be (and is likely) due to unrelated stars, rather than the lens itself. The presence (or lack) of any blend flux in MOA-II data therefore provides no diagnostic power on the boundedness of planetary lenses. Consequently, Sumi et al. (2011) were only able to look for evidence of a host lens in their 10 short-timescale events through the first two channels and so we do not need to consider the third channel in this study.

Detection of either a primary bump or anomalies due to the planetary caustic depends on the geometry of the event and the quality of the observations (e.g. total number of observations, cadence, photometric precision). For a given set of observational (i.e. survey) parameters, the fractions of events due to wide-separation planets for which the presence of a primary is expected to be detected by these two different channels scales as $\sim s^{-1}$ and $\sim s^{-2}$, respectively. In this section, we provide brief descriptions of these two channels and how we implement them in this study, but for a more in-depth look at distinguishing events due to wide-separation and free-floating 
planets, see Han et al. (2005) and references therein.

\subsection{Low-Magnification Primary Bumps}

The source trajectory in some fraction of planetary microlensing events, $\mathcal{W}(s)$, will be such that the impact parameter to the primary, $u_{\star}$, is small enough that it will produce a detectable magnification. For a given observational cadence and signal-to-noise ratio $(\mathrm{S} / \mathrm{N} ; \mathcal{Q})$ threshold, this fraction depends solely on the geometry of the lens system and has the form

$$
\mathcal{W}(s)= \begin{cases}1, & s \leq u_{\star, \mathrm{th}}, \\ \frac{2}{\pi} \sin ^{-1}\left(\frac{u_{\star, \mathrm{th}}}{\hat{s}}\right), & s>u_{\star, \mathrm{th}},\end{cases}
$$

where $\hat{s} \equiv s-1 / s$ is the projected separation of the center of the planetary caustic from the host star and $u_{\star, \text { th }}$ is the maximum source impact parameter to the primary in units of the primary Einstein radius, $\theta_{\mathrm{E}}$, such that the primary bump is just detectable. The form for the maximum impact parameter we adopt is given by Han et al. (2005) as

$$
\begin{aligned}
u_{\star, \mathrm{th}}= & 2.2\left(\frac{M_{\mathrm{L}}}{0.3 M_{\odot}}\right)^{1 / 14}\left(\frac{f_{\mathrm{obs}}}{50 \text { day }^{-1}}\right)^{1 / 7} \times \\
& \left(\frac{\sigma_{\mathrm{p}}}{0.05}\right)^{-2 / 7}\left(\frac{\mathcal{Q}_{\mathrm{th}}}{80}\right)^{-2 / 7},
\end{aligned}
$$

where $M_{\mathrm{L}}$ is the primary lens mass, $f_{\text {obs }}$ is the frequency of observations, $\sigma_{\mathrm{p}}$ is the fractional photometric precision of each observation, and $\mathcal{Q}_{\text {th }}$ is the $\mathrm{S} / \mathrm{N}$ threshold for detection. The values to which we have scaled this relation are set by the selection criteria of the Sumi et al. (2011) study and are typical for MOA-II data (T. Sumi, private communication). For the given survey parameters $\left(f_{\text {obs }}, \sigma_{\mathrm{p}}\right.$, and $\left.\mathcal{Q}_{\mathrm{th}}\right)$ and at fixed primary lens mass $\left(M_{\mathrm{L}}\right)$, we find that $\mathcal{W}(s)=1$ out to projected separations $s \lesssim 2.6$. In the limit of large projected separations, $s \gg 1$, the probability of detecting the primary through this channel falls off as $s^{-1}$. We note that $u_{\star, \text { th }}$ is only weakly dependent on $f_{\mathrm{obs}}, M_{\mathrm{L}}$, and $\sigma_{\mathrm{p}}$ and thus argue that our approximation that these parameters are the same for all events is reasonable. Figure 1 shows a plot of $\mathcal{W}(s)$ and illustrates the that, for parameters typical of the MOA-II survey and the selection criteria set by Sumi et al. (2011), this is not the primary channel for detecting signatures of the host lens except at separations beyond $s \gtrsim 10(a \gtrsim 28 \mathrm{AU})$.

\subsection{Anomalies Due to the Planetary Caustic}

The rate of planetary events where signatures of the primary due to anomalies near the peak of the light curve arising from the source passing near (or crossing) the planetary caustic relative to the total rate of planetary microlensing events is

$$
\mathcal{R}_{\mathrm{pc}}(q, s)=\frac{u_{\mathrm{pl}, \max }(s)}{\bar{u}_{\mathrm{S} 11}},
$$

where $u_{\mathrm{pl}, \max }(s)$ is the maximum required impact parameter for signatures of the planetary caustic to be just detectable as a function of the projected separation, $s$, and $\bar{u}_{\mathrm{S} 11}=0.153$ is the median impact parameter measured by Sumi et al. (2011) for the 10 short-timescale

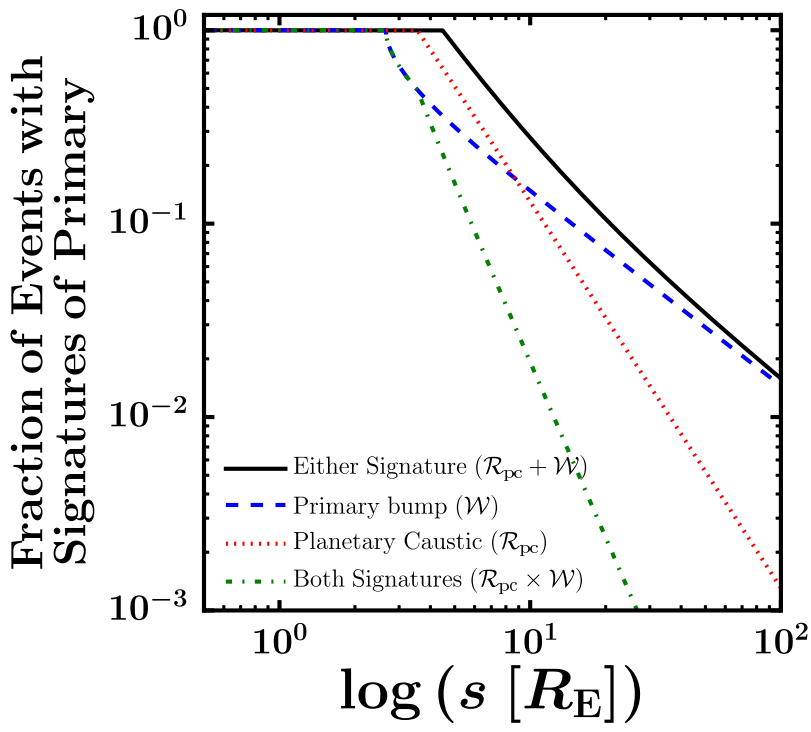

Figure 1. Fraction of planetary microlensing events for which signatures of primary are expected to be detectable, i.e., the fraction of planetary events we can distinguish as being due to bound planets rather than free-floating planets (assuming $M_{\mathrm{L}}=0.3 M_{\odot}$, $f_{\text {obs }}=50$ day $^{-1}, \sigma_{\mathrm{p}}=0.05$, and $\left.\mathcal{Q}_{\mathrm{th}}=80\right)$. We examine two channels for detecting signatures of the primary: 1) relatively longtimescale, low-magnification primary bump, and 2) anomalies near the peak of the light curve due to the source passing near (or crossing) the planetary caustic. The relative probabilities of these channels fall off with increasing projected separation as $\mathcal{W}(s) \sim s^{-1}$ and $\mathcal{R}_{\mathrm{pc}} \sim s^{-2}$, respectively. See text for a more detailed description.

$\left(0.3 \leq t_{\mathrm{E}} /\right.$ day $\left.\leq 3\right)$ events in their sample. If the MOAII survey were uniformly sensitive to events with respect to impact parameter, then we would have chosen to normalize $\mathcal{R}_{\mathrm{pc}}$ by $u_{0}=1$, the maximum impact parameter allowed by the criteria set by Sumi et al. (2011), with which they selected their sample (see Section 2 of the supplemental materials of Sumi et al. 2011). In reality, there is a bias towards smaller impact parameters (since the total magnification, $A$, depends on the lens-source projected separation, $u$, as $\left.A(u)=\left[\left(u^{2}+2\right) /\left(u \sqrt{u^{2}+4}\right)\right]\right)$ and we therefore attempt to account for this by normalizing $\mathcal{R}_{\mathrm{pc}}$ by $\bar{u}_{\mathrm{S} 11}$.

We assume that in order for signatures of the planetary caustic to be detectable in the light curve that $u_{\mathrm{pl}, \max } \sim \theta_{\mathrm{c}}$, where $\theta_{\mathrm{c}}$ is the angular radius of the planetary caustic, which we assume to be circular in shape with a size given by the height of the caustic in the direction perpendicular to the star-planet axis. Adapting equation 9 of $\mathrm{Han}(2006)$ to be consistent with our adopted geometry, we find the following expression for $\theta_{c}$ (which has units of the primary Einstein radius)

$$
\theta_{\mathrm{c}}=\frac{2}{s \sqrt{s^{2}+1}}
$$

There is a projected separation, $s_{\mathrm{c}}$, at which $\theta_{\mathrm{c}}>\bar{u}_{\mathrm{S} 11}$ and interior to which $\mathcal{R}_{\mathrm{pc}}$, as defined by equation (3), becomes greater than unity. This works out to be $s_{\mathrm{c}} \simeq 3.5$, which roughly corresponds to a projected separation in physical units of $r_{\perp, \mathrm{c}} \approx 10 \mathrm{AU}$ for typical event parameters and a semimajor axis of $a_{\mathrm{c}} \approx 12 \mathrm{AU}$ for the median projection angle of a circular orbit. Thus, to ensure that 
$\mathcal{R}_{\mathrm{pc}} \leq 1$, we adopt the definition

$$
u_{\mathrm{p} 1, \max }(s)= \begin{cases}\bar{u}_{\mathrm{S} 11}, & s \leq s_{\mathrm{c}}, \\ \theta_{\mathrm{c}}, & s>s_{\mathrm{c}},\end{cases}
$$

and equation (3) takes the form

$$
\mathcal{R}_{\mathrm{pc}}(q, s)= \begin{cases}1, & s \leq s_{\mathrm{c}}, \\ \frac{\theta_{\mathrm{c}}}{\bar{u}_{\mathrm{S} 11}}, & s>s_{\mathrm{c}} .\end{cases}
$$

In doing so, we are effectively assuming that if a planetary event with $s \leq s_{\mathrm{c}}$ is detected, anomalies due to the planetary caustic will always be detected. For our purposes, this is not a problem, since we are only concerned with computing the fraction of events for which we expect to see evidence of a primary (regardless of the exact channel). Figure 1 illustrates our expectation that for $s \lesssim 10$, perturbations due to the planetary caustic are the primary channel for revealing the presence of a host star.

\section{METHODOLOGY}

In Clanton \& Gaudi (2016), we performed a joint analysis of results from five different surveys for exoplanets employing three independent discovery techniques: microlensing (Gould et al. 2010; Sumi et al. 2010), radial velocity (specifically, the long-term trends; Montet et al. 2014), and direct imaging (Lafrenière et al. 2007; Bowler et al. 2015). We found that the results of all these surveys can be simultaneously explained by a single population of planets described by a with a simple, joint power-law distribution in mass and semimajor axis given by

$$
\frac{d^{2} N_{\mathrm{pl}}}{d \log m_{p} d \log a}=\mathcal{A}\left(\frac{m_{p}}{M_{\mathrm{Sat}}}\right)^{\alpha}\left(\frac{a}{2.5 \mathrm{AU}}\right)^{\beta} .
$$

This model has just four free parameters, $\left\{\alpha, \beta, \mathcal{A}, a_{\text {out }}\right\}$, where $a_{\text {out }}$ is the outer cutoff radius of the semimajor axis distribution. The median values and $68 \%$ confidence intervals we infer for these parameters are summarized in Table 1. Note that the quoted uncertainties, particularly those on $\beta$ and $a_{\text {out }}$, are correlated (see Figures 25-27 in Clanton \& Gaudi 2016). In this paper, we employ this population of bound planets that is known to be consistent with microlensing, RV (long-term trend detections), and direct imaging surveys to explain (at least a significant fraction) of the overabundance of short-timescale microlensing events observed in the MOA-II data, and thus derive constraints on the frequency of truly freefloating planets in the Galaxy.

We first sample the posterior distributions (including covariances) derived in Clanton \& Gaudi (2016) to obtain parameters (i.e. $\alpha, \beta, \mathcal{A}$, and $a_{\text {out }}$ ) for a random population of (bound) planets, and draw an ensemble of planets from the resultant distribution function. We then generate a corresponding set of simulated microlensing events, precisely following the procedure we outline in Clanton \& Gaudi (2014a, b, 2016), but with a slightly altered LMF. In this paper, we adopt "Model 1" exactly as it is presented in Sumi et al. (2011), which includes populations of brown dwarfs, main-sequence stars, white dwarfs, neutron stars, and black holes that are described by power-law distributions in their initial mass. We fix the slope of the LMF in the brown dwarf regime to be the median value reported by Sumi et al. (2011), as the inferred value for this slope is not significantly different when the fitting the full timescale distribution versus fitting the timescale distribution for $t_{E}>2$ days (which we verified with our own, completely independent, fitting procedures). We display a plot of the initial lens mass distribution (relevant for the remnant populations) in Figure 2 along with plots of the final LMF weighted by number, mass, and contribution to the microlensing event rate along a given line of sight. The relative numbers of brown dwarfs, main-sequence stars, white dwarfs, neutron stars, and black holes by number, mass, and event rate are (38:52:9.5:1.1:0.16), (5.9:63:22:5.7:3.2), and (17:63:17:2.9:0.84), respectively (consistent with Gould 2000). We find, as did Sumi et al. (2011), that the numbers of brown dwarfs, white dwarfs, neutron stars, and black holes relative to main-sequence stars are (73:18:2.1:0.31).

Figure 3 shows a plot of the predicted distribution of timescales for the brown dwarf, main-sequence, and remnant lenses in our simulated sample against the observed distribution. Our predicted distribution has been corrected for the detection efficiency determined by Sumi et al. (2011) (shown in Figure S2 of their supplementary materials) and normalized such that the total number of simulated microlensing events matches that of the observed sample. Note that this is not a fit to the observed distribution, but rather, it is a prediction based on a fit performed by Sumi et al. (2011) that we use to fix the slope of the LMF in the brown dwarf regime. By eye, this appears to be a good match for events with $t_{E}>2$ days (providing a degree of confidence in our adopted Galactic model and LMF), but the overabundance of shorter-timescale events in the observed distribution is clear. We will attempt to explain these shorttimescale events with bound planetary companions for which we do not expect to see evidence of a primary in the microlensing light curves.

Having generated a population of planets with corresponding microlensing events as described above, we then determine the probability that the primary (i.e. host star) would not be detected in each event given the survey parameters of MOA-II, $P_{\star}^{\prime}=[1-\mathcal{W}(s)] \times$ $\left[1-\mathcal{R}_{\mathrm{pc}}(q, s)\right]$, where $\mathcal{W}(s)$ is the fraction of events where a low-magnification primary bump is expected to be detectable and $\mathcal{R}_{\mathrm{pc}}(q, s)$ is the fraction of events where perturbations in the light curve due to the planetary caustic are expected (see Section 3 for the formal definitions and a discussion of these quantities). We then construct the predicted timescale distribution for the combination of our adopted LMF and the associated population of bound planets that appear to be freefloating, again taking care to correct for the detection efficiency of MOA-II to events as a function of $t_{E}$. This predicted timescale distribution serves as our likelihood function (for which there is no analytic form). We calculate the likelihood of a given planet population by applying this numerically-generated likelihood function to the individual measurements of $t_{E}$ for each of the 474 events comprising the observed distribution presented in Sumi et al. (2011). These data are published in Table 4 of Sumi et al. (2013). We repeat this procedure for all 
Table 1

Median values and $68 \%$ uncertainties inferred by Clanton \& Gaudi (2016) for the parameters of a population of planets that is consistent with results from the microlensing surveys of Gould et al. (2010) and Sumi et al. (2010), the Gemini Deep Planet Survey (Lafrenière et al. 2007) and Planets Around Low Mass Stars (Bowler et al.|2015) direct imaging surveys, and the CPS TRENDS (Montet et al. 2014) RV survey.

\begin{tabular}{c||c|c|c|c}
\hline \multicolumn{1}{c||}{ Planet Evolutionary } & \multicolumn{4}{c}{ Median Values and $68 \%$ Uncertainties } \\
Model & $\alpha$ & $\beta$ & $\mathcal{A}\left[\mathrm{dex}^{-2}\right]$ & $a_{\text {out }}[\mathrm{AU}]$ \\
\hline $\begin{array}{c}\text { "Hot-Start" } \\
\text { (Baraffe et al. 2003) }\end{array}$ & $-0.86_{-0.19}^{+0.21}$ & $1.1_{-1.4}^{+1.9}$ & $0.21_{-0.15}^{+0.20}$ & $10_{-4.7}^{+26}$ \\
\hline $\begin{array}{c}\text { "Cold-Start" } \\
\text { (Fortney et al. 2008) }\end{array}$ & $-0.85_{-0.19}^{+0.21}$ & $1.1_{-1.3}^{+1.9}$ & $0.21_{-0.15}^{+0.20}$ & $12_{-6.2}^{+50}$ \\
\hline \hline
\end{tabular}
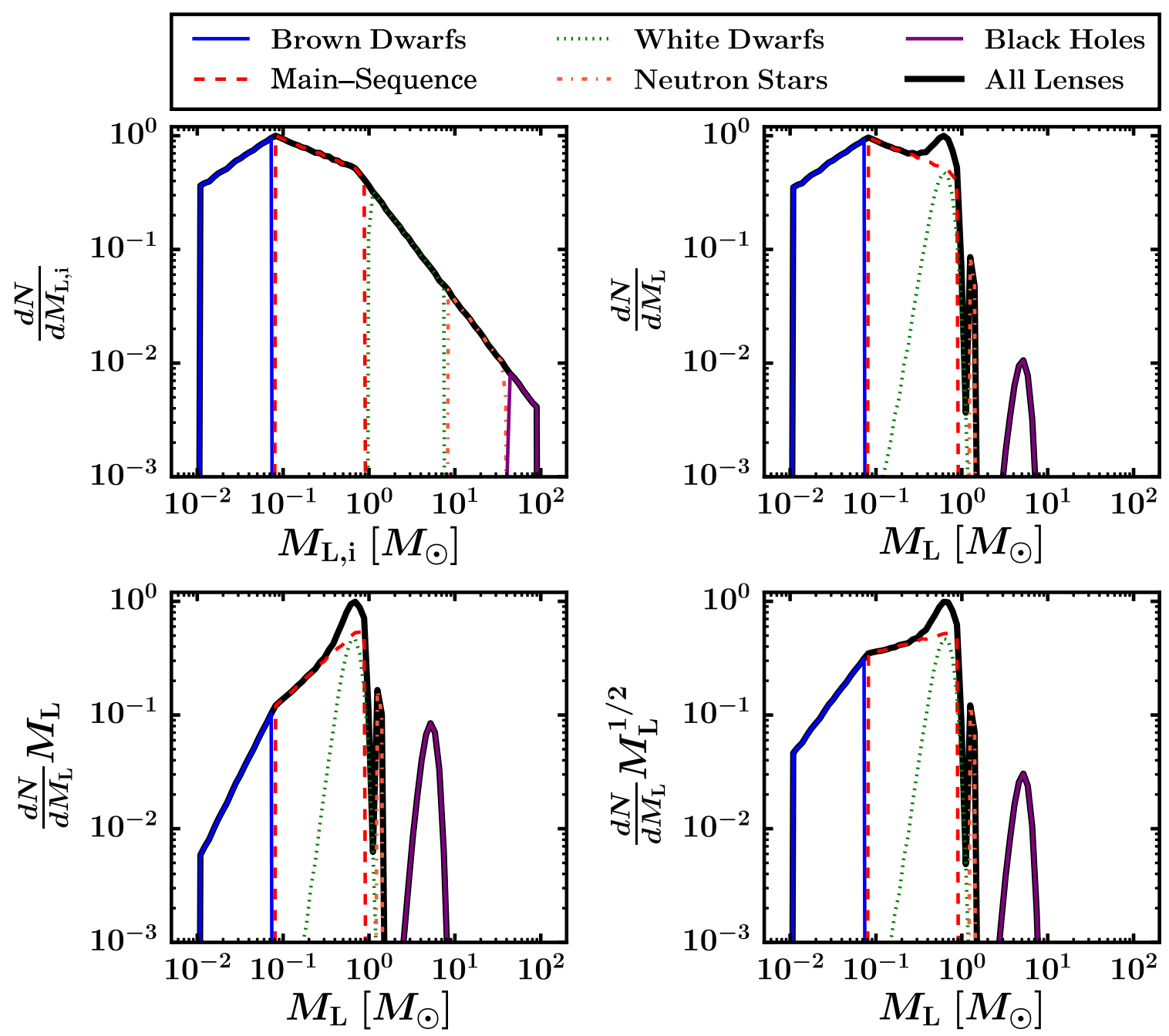

Figure 2. The lens mass function we adopt in this study (identical to "Model 1" of Sumi et al. 2011), consisting of populations of brown dwarfs, main-sequence stars, white dwarfs, neutron stars, and black holes, each of which is described by a power-law distribution in their initial mass. The top left panel plots the initial lens mass function, and the top right panel plots the final lens mass function. The bottom left and bottom right panels show the final mass function, weighted by lens mass and event rate $\left(\Gamma \propto M_{L}^{1 / 2}\right.$ along a given sight line and at fixed $D_{l}, D_{s}$, and $\boldsymbol{\mu}_{\text {rel }}$ ), respectively.

planet populations Clanton \& Gaudi (2016) found to be consistent with radial velocity, microlensing, and direct imaging surveys. This allows us to place constraints on the fraction of short-timescale $\left(t_{E}<2\right.$ days $)$ microlensing events due to free-floating planets. In order to determine an actual number of such planets (e.g. relative to main-sequence stars), we must adopt an ad hoc form for the mass function of free-floating planets. Therefore, our estimate of the number of free-floating planets per star is less robust (i.e. more model dependent) than our estimate of the fraction of short-timescale events due to free-floating planets. We present and discuss our results and main sources of uncertainty in the following section.

\section{RESULTS AND DISCUSSION}

Figure 4 shows the best-fit (i.e. maximum likelihood) expected timescale distribution for the combination of our canonical LMF described in the previous sec- 


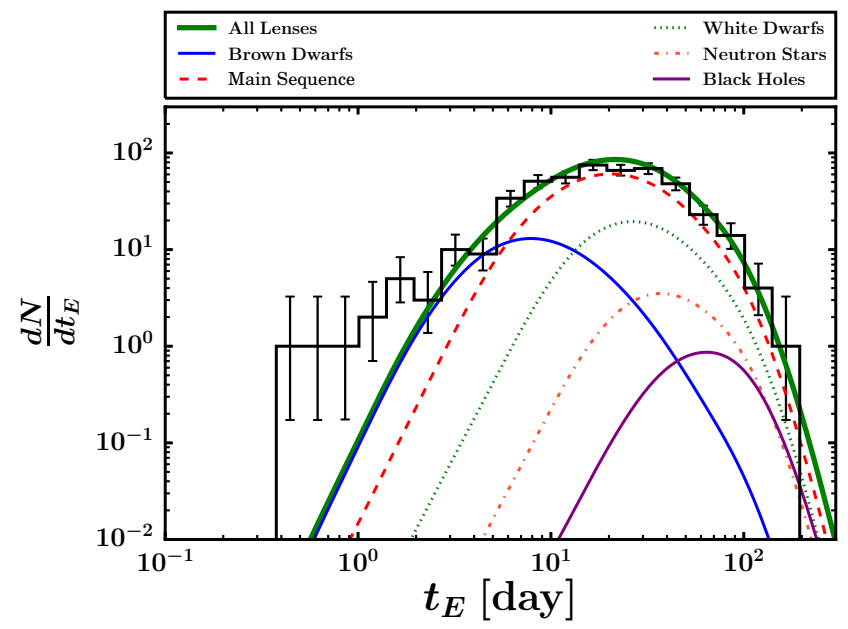

Figure 3. Predicted timescale distribution for populations of brown dwarfs, main-sequence stars, and stellar remnants (colored lines) and the observed timescale distribution reported by Sumi et al. (2011) (black histogram). The predicted timescale distribution has been subjected to the measured detection efficiency of the Sumi et al. (2011) survey and normalized to the total number of observed microlensing events. The number of short-timescale microlensing events $\left(t_{E} \leq 2\right.$ days $)$ predicted by our adopted LMF is 1.1, compared to the observed number of 10, demonstrating a clear overabundance of such short-timescale events in the observed sample.

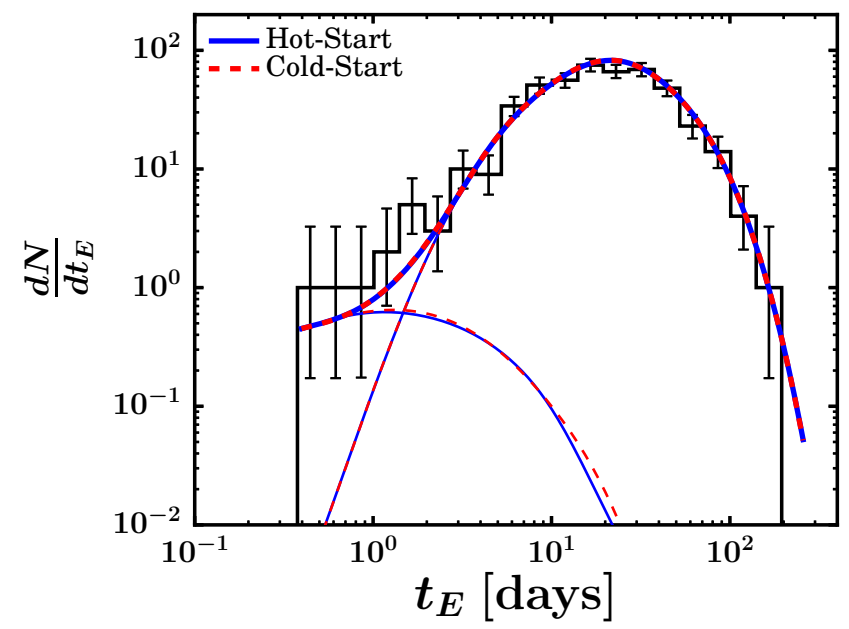

Figure 4. Maximum likelihood fits to the observed timescale distribution (black histogram; Sumi et al. 2011) for our canonical LMF and a population of bound, wide-separation planets that is consistent with results from radial velocity, microlensing, and direct imaging surveys (Clanton \& Gaudi 2016), assuming either "hot-start" (blue lines; Baraffe et al. 2003) or "cold-start" (red lines; Fortnev et al. 2008) planet evolutionary models. The thick lines show the expected timescale distribution from all lenses, while the thin lines show the expected contributions from planets (the curves peaking at shorter timescales) and brown dwarfs, main-sequence stars, and remnants (the curves peaking at longer timescales). For these maximum likelihood fits, wide-separation, bound planets account for roughly 2.9 of the 10 observed shorttimescale $\left(t_{E}<2\right.$ days $)$ events in both the "hot-start" and "coldstart" cases, and brown dwarfs account for about one event.

tion with populations of wide-separation, bound planets found by Clanton \& Gaudi (2016) to be consistent with results from radial velocity, microlensing, and direct imaging surveys for either "hot-start" (Baraffe et al. 2003) or "cold-start" (Fortney et al. 2008) planet evolutionary models. Given that the parameters of these planet populations (i.e. the slopes of the mass function, $\alpha$ and semimajor axis function, $\beta$, normalizations, $\mathcal{A}$, and outer cutoff radii, $a_{\text {out }}$ ) for the "hot-start" and "cold-start" models are not too different (see Section 5.2 of Clanton \& Gaudi 2016), it is not surprising that the fits for these different models shown in Figure 4 are so similar. The parameter values for the best-fit "hotstart" planet population are $\alpha=-0.85, \beta=0.091$, $\mathcal{A}=0.26 \mathrm{dex}^{-2}$, and $a_{\text {out }}=740 \mathrm{AU}$, and those for the best-fit "cold-start" population are similar. The value of $a_{\text {out }}$ for this best fit is quite large due to the fact that the number of planets for which we do not expect to see signatures of a primary lens (host star) in the microlensing light curves (which are needed to explain the overabundance of short-timescale events) increases with this outer cutoff radius. For large $a_{\text {out }}$, planets are allowed to be in very wide-separation orbits which lead to smaller planetary caustic sizes (and thus small rates of planetary caustic events, since at fixed $q, R_{\mathrm{pc}} \sim \theta_{c} \propto s^{-2}$ for $s \gg 1$ ) and which have low probability for source trajectories that pass near the primary $\left(\propto s^{-1}\right.$ for $\left.s \gg 1\right)$. However, in order for a planet population with a large value of $a_{\text {out }}$ to be consistent with the non-detections from direct imaging surveys (i.e. Lafrenière et al. 2007 and Bowler et al. 2015), the slope of the semimajor axis distribution function must be shallow, and indeed, the best-fit population has $\beta$ near zero (corresponding to Öpik's law; Opik 1924).

In Figure 5, we display the best-fit to the observed timescale distribution along with the range of fits in the $68 \%$ confidence interval. It is clear from this figure that while we can explain some fraction of the shorttimescale events with bound, wide-separation planets, an overabundance remains (particularly at timescales between 1-2 days). This suggests that either our assumed planet population model is incorrect in regions of parameter space where we currently have no observational constraints $\left(m_{p} \lesssim M_{\text {Jup }}\right.$ at separations $\left.a \gtrsim 10 \mathrm{AU}\right)$, or free-floating planets are responsible for the remaining short-timescale events. We have no way of testing the former, but for the latter case we can constrain the fraction of short-timescale events that would be due to free-floating planets given our assumed planet model.

For each planet population we fit to the observed timescale distribution, we determine the number of residual events with $0.3<t_{E}$ /days $<2$ and divide by the number of observed events in this same range of $t_{E}$ to compute the fraction of such events which are expected to be due to free-floating planets, $f_{\mathrm{ff}}$. We plot the posterior distribution of $f_{\mathrm{ff}}$ in Figure 6 and report the corresponding median values, $68 \%$, and $95 \%$ confidence intervals in Table 2. The posterior for the "cold-start" case is shifted slightly towards lower $f_{\mathrm{ff}}$, as expected, but it is not significantly different from the "hot-start" case.

In order to turn this fraction, $f_{\mathrm{ff}}$, into an actual number of free-floating planets (relative to main-sequence stars, for example), we must assume a form for their mass function. To this end, we assume that the freefloating planet mass function is given by a Dirac delta function, $\delta\left(m_{\mathrm{p}, \text { ff }} / M_{\mathrm{Jup}}-2\right)$. We chose to center the delta function at $2 M_{\mathrm{Jup}}$ as such a free-floating planet population lead to a timescale distribution that most closely matches (by eye) the residuals obtained from subtract- 
Table 2

Median values, $68 \%$, and $95 \%$ confidence intervals on both the fraction of short-timescale events due to free-floating planets, $f_{\text {ff }}$, and the number of free-floating planets relative to main-sequence stars, $N_{\mathrm{ff}}$. We report these values for our analyses that assume either "hot-start" (Baraffe et al. 2003) or "cold-start" (Fortney et al. 2008) planet evolutionary models.

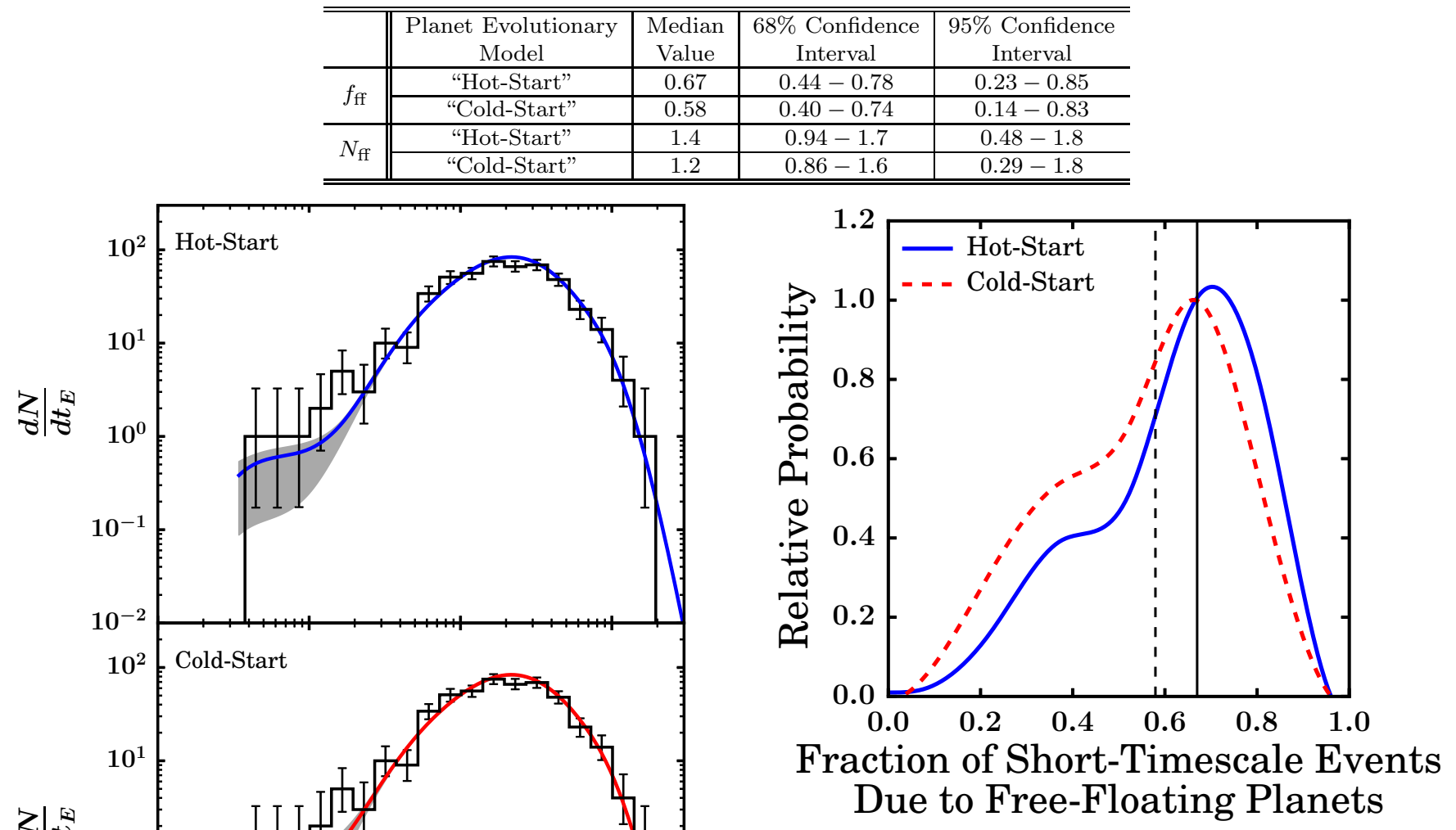

Figure 6. The fraction of short-timescale $\left(t_{E}<2\right.$ days $)$ microlensing events that must be due to free-floating planets, $f_{\mathrm{ff}}$, for our analyses that assume either "hot-start" (blue; Baraffe et al. 2003) or "cold-start" (red; Fortnev et al. 2008) planet evolutionary models. The vertical, black lines mark the median values of these posterior distributions.

delta-function mass distribution at $2 M_{\text {Jup }}$ in Figure 8 , and we show the range of fits in the $68 \%$ confidence interval in Figure 9 .

The median number of free-floating planets per main-

Figure 5. Maximum likelihood and $68 \%$ confidence interval fits to the observed timescale distribution (black histograms; Sumi et al. 2011) for our canonical LMF and a population of bound, wideseparation planets that is consistent with results from radial velocity, microlensing, and direct imaging surveys (Clanton \& Gaudi) 2016), assuming either "hot-start" (top panel; Baraffe et al. 2003) or "cold-start" (bottom panel; Fortney et al. 2008) planet evolutionary models.

ing off our LMF and the population of wide-separation, bound planets as described in the previous section. Admittedly, this is a rough calculation, however given the level of precision of this study, we do not believe a more careful analysis is currently warranted (especially given the fact that we currently have no constraints on the actual form of the free-floating planet mass function that we must adopt). The resultant posterior on the number of free-floating planets per main-sequence star is plotted in Figure 7 and the corresponding median values, $68 \%$, and $95 \%$ confidence intervals are reported in Table 2. We plot the maximum likelihood fits for the "hot" and "cold-start" analyses, including the contribution from free-floating planets, under this assumption of a sequence star we find, $N_{\mathrm{ff}}=1.4_{-0.46}^{+0.30}\left(N_{\mathrm{ff}}=1.2_{-0.34}^{+0.40}\right)$ for the "hot-start" ("cold-start") case, is quite a large number that seems difficult to explain with any known formation mechanism (see Section 1 for discussion on the formation channels for free-floating planets). However, more "comfortable" values of $N_{\mathrm{ff}}=0.48\left(N_{\mathrm{ff}}=0.29\right.$; "cold-start") are allowed to within $95 \%$ confidence and could perhaps be easier to explain. Furthermore, these values are sensitive to a number of assumptions, most notably the free-floating planet mass function and the model for the population of wide-separation, bound planets. The remainder of this section is devoted to discussion of these two primary sources of uncertainty.

Free-Floating Planet Mass Function: Without direct lens mass measurements for each of the short-timescale events, the only constraining power currently available on the free-floating planet mass function is, in fact, contained in the observed microlensing event timescale distribution. In order to constrain the free-floating planet mass function using the timescale distribution, prior knowledge of which of the short-timescale events are ac- 


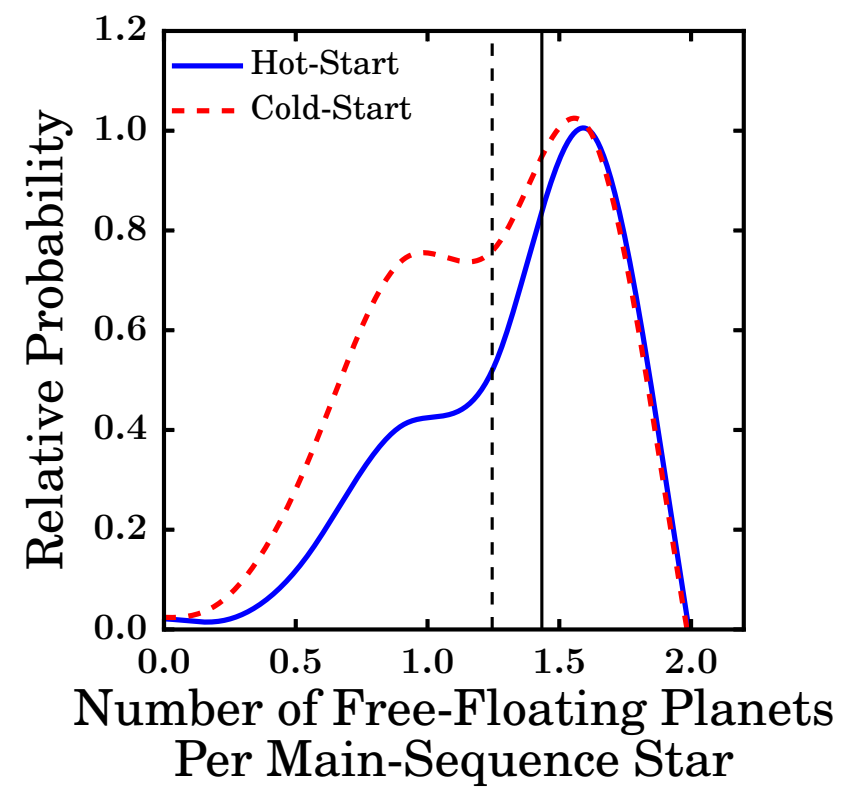

Figure 7. The number of free-floating planets per main-sequence star, $N_{\mathrm{ff}}$, required to explain the residual short-timescale $\left(t_{E}<\right.$ 2 days) microlensing events after fits of our canonical LMF and populations of wide-separation, bound planets are subtracted for our analyses that assume either "hot-start" (blue; Baraffe et al. 2003) or "cold-start" (red; Fortney et al. 2008) planet evolutionary models. The vertical, black lines mark the median values of these posterior distributions. Estimating this quantity requires an assumption about the mass function of free-floating planets. Here, we have chosen a delta function at a mass of $2 M_{\mathrm{Jup}}$ (see text for discussion).

tually due to free-floating planets would be required. With such knowledge, one could perform a model comparison of fits to the observed timescale distribution assuming different forms for the free-floating planet mass function. Unfortunately, we do not know exactly which events are caused by truly unbound planets (due to fundamental degeneracies that affect a majority of microlensing observations; see Gaudi 2012 and references therein) and the number of short-timescale $t_{E} \lesssim 2$ days is small, making such a study difficult. This paper is an attempt to address the first of these issues by simulating microlensing events of wide-separation, bound planets to determine (statistically) the fraction of the shorttimescale events that are caused by free-floating planets. Of course, the results presented herein are therefore dependent on our assumed model of bound planets.

Data from ongoing and future microlensing surveys will allow direct measurements of both the frequency and mass function of free-floating planets, as well as their spatial distribution within our Galaxy. The recent K2 Campaign 9 (K2C9) consisted of a survey toward the Galactic bulge (Henderson et al. 2015). For short-timescale microlensing events observed simultaneously from Kepler and ground-based observatories (such that we see two distinct source trajectories), it is possible (for some events) to directly measure the lens mass and distance and obtain better constraints on the existence of a primary (i.e. host star) since Kepler provides precise, continuous observations (see Henderson et al. 2015; Henderson \& Shvartzvald 2016). However, given the short, $\sim 80$ day duration of K2C9, the sample size will likely be too small to make population-level inferences

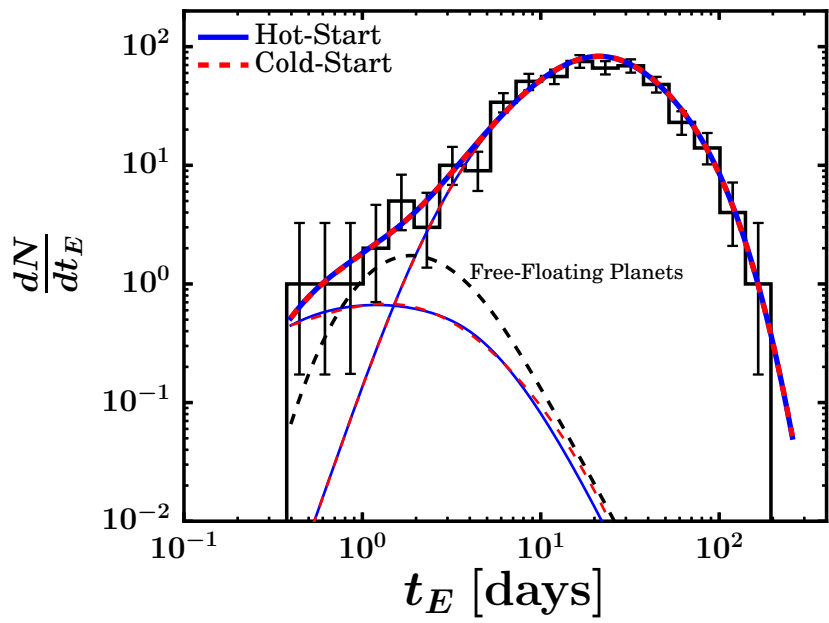

Figure 8. Maximum likelihood fits to the observed timescale distribution (black histogram; Sumi et al., 2011) for our canonical $\mathrm{LMF}$, a population of bound, wide-separation planets that is consistent with results from radial velocity, microlensing, and direct imaging surveys (Clanton \& Gaudi 2016), assuming either "hotstart" (blue lines; Baraffe et al. 2003) or "cold-start" (red lines; Fortney et al. 2008) planet evolutionary models, and a population of free-floating planets whose mass function is a $\delta$ function at $2 M_{\text {Jup }}$ (black dashed line). The thick lines show the expected timescale distribution from all lenses, while the thin lines show the expected contributions from bound planets (the curves peaking at shorter timescales) and brown dwarfs, main-sequence stars, and remnants (the curves peaking at longer timescales). For these maximum likelihood fits, wide-separation, bound planets account for roughly 2.9 of the 10 observed short-timescale $\left(t_{E}<2\right.$ days events in both the "hot-start" and "cold-start" cases, brown dwarfs account for about one event, and free-floating planets make up the difference.

about free-floating planets other than (at least limits) on their occurrence rates (recall that the event rate scales as $\Gamma \propto M_{L}^{1 / 2}$ ). Indeed, Penny et al. (2016) predict that K2C9 will detect between 1.4 and 7.9 microlensing events due to free-floating planets (assuming 1.9 free-floating planets per main-sequence star per the Sumi et al. 2011 result). Of these expected detections, Penny et al. (2016) predict that for between 0.42 and 0.98 it will be possible to gain a complete solution (i.e. to measure both finitesource effects and microlens parallax). Given the results we present in this paper, these numbers would be smaller by a factor of $\sim 0.6$ (refer to Table 22), and thus it is unlikely K2C9 will actually directly measure the lens mass in a short-timescale event.

Fortunately, the microlensing survey of the WideField InfraRed Survey Telescope (hereafter WFIRST; Spergel et al. 2015) will ultimately, when combined with ground-based observations, provide the necessary data to directly measure frequencies, masses, and distances for a large sample of free-floating planets with masses down to that of Mars (see Gould et al. 2003 and Yee 2013, who demonstrate that simultaneous observations from the ground and WFIRST at L2 will enable the measurement of the parallax of planetary events). Depending on the exact occurrence rates, WFIRST will detect hundreds to $\sim$ thousands of free-floating planets (see Table 2-6 of Spergel et al. 2015).

The Population of Wide-Separation, Bound Planets: The model we assume in this paper is a joint powerlaw distribution function in planet mass and semimajor axis that Clanton \& Gaudi (2016) demonstrate to be 


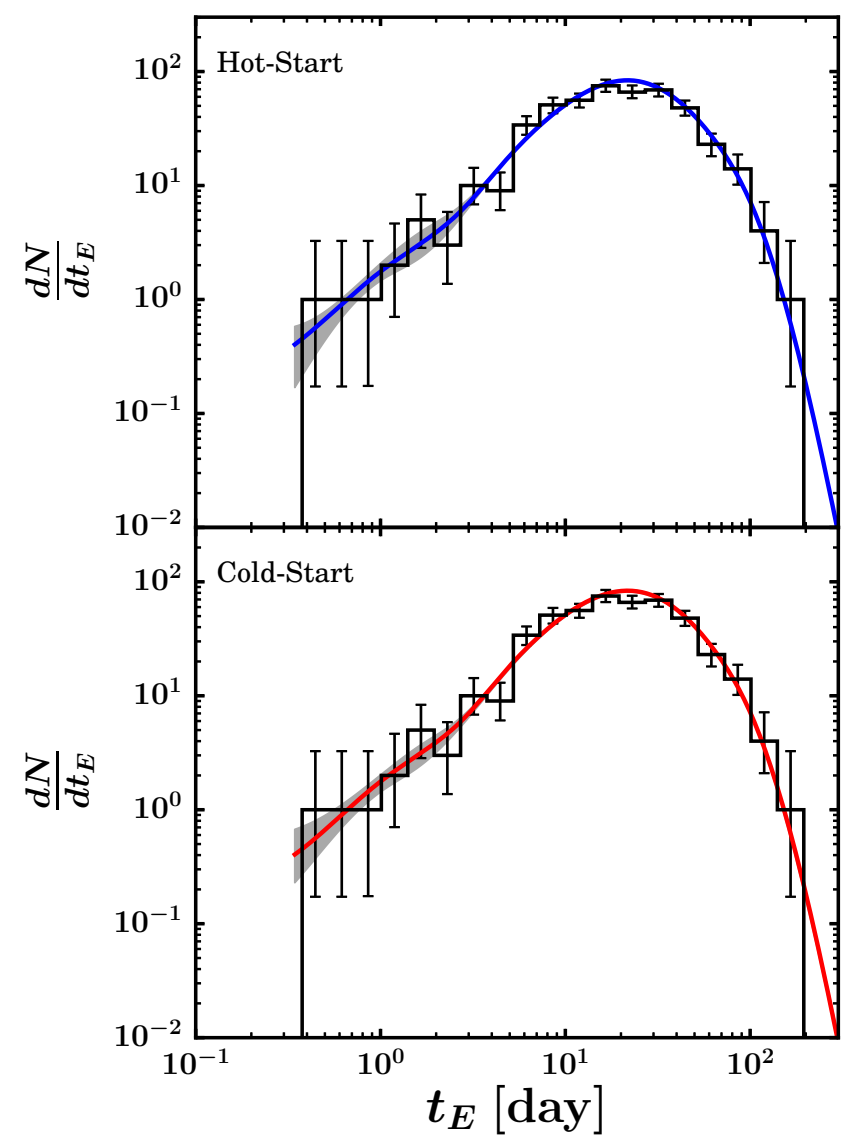

Figure 9. Maximum likelihood and $68 \%$ confidence interval fits to the observed timescale distribution (black histograms; Sumi et al. 2011) for our canonical LMF, a population of bound, wideseparation planets that is consistent with results from radial velocity, microlensing, and direct imaging surveys (Clanton \& Gaudi 2016), assuming either "hot-start" (top panel; Baraffe et al. 2003) or "cold-start" (bottom panel; Fortney et al. 2008) planet evolutionary models, and a population of free-floating planets whose mass function is a $\delta$ function at $2 M_{\mathrm{Jup}}$.

consistent with results from radial velocity, microlensing, and direct imaging surveys (the caveats and uncertainties of which are laid bare in Section 6 of Clanton \& Gaudi 2016). However, the region of planet parameter space we examine in this paper $\left(m_{p} \lesssim M_{\text {Jup }} ; a \gtrsim 10 \mathrm{AU}\right)$ is not directly constrained by any observations. We have implicitly assumed that our distribution function extrapolates into this region of parameter space. It could be the case that the form of the planet mass function depends on semimajor axes for $a \gtrsim 10$ AU, which could significantly alter our conclusions. For example, if no planets with masses $m_{p} \gtrsim M_{\text {Jup }}$ form beyond $\sim 10$ AU but there is an abundance of slightly less massive planets, we could easily explain most, if not all, the short-timescale microlensing events with bound planets and still satisfy results from all radial velocity, microlensing, and direct imaging surveys of M stars.

Future observations will provide the necessary sensitivity to test the planetary mass function at wideseparations and determine whether or not the mass function measured by microlensing surveys extends further out (as we have assumed to be the case in this paper). The James Webb Space Telescope (JWST; Gardner et al. 2006) is expected to have the capability to achieve contrasts of $\sim 10^{-5}$ at angular separations $\gtrsim 0.6$ arcseconds for observations at $\sim 4.5 \mu \mathrm{m}$ with NIRCam (and even greater sensitivity at larger separations; Horner \& Rieke 2004; Krist et al. 2007). A survey of nearby, young M stars with $J W S T /$ NIRCam as proposed by Schlieder et al. (2016) has the potential to probe down to masses of $\sim 0.1 M_{\text {Jup }}$ at separations of $\sim 10 \mathrm{AU}$, complementary (and perhaps with some overlap) to microlensing surveys.

\section{SUMMARY}

In this paper, we attempt to explain the observed overabundance of short-timescale $\left(t_{E}<2\right.$ days $)$ microlensing events with populations of wide-separation, bound exoplanets that are known to be simultaneously consistent with results from radial velocity, microlensing, and direct imaging surveys. We select planetary systems from such populations that we (statistically) expect not to show evidence of a primary (i.e. host star) in their microlensing light curves, either via low-magnification bumps or anomalies near the peak of the light curve due to close approaches to, or crossings of, the planetary caustics. We fit the observed timescale distribution reported by Sumi et al. (2011) with these planetary systems and a primary lens mass function consisting of brown dwarfs, main-sequence stars, white dwarfs, neutron stars, and black holes. We find that wide-separation, bound planets can explain some of the short-timescale events, but (assuming our joint power-law planet distribution function in mass and semimajor axis presented in Clanton \& Gaudi 2016 is correct) free-floating planets must account for a fraction of the short-timescale events of either $f_{\mathrm{ff}}=0.67(0.23-0.85$ at $95 \%$ confidence $)$ for "hot-start" planet evolutionary models (Baraffe et al. $2003)$ or $f_{\mathrm{ff}}=0.58(0.14-0.83$ at $95 \%$ confidence $)$ for "cold-start" models (Fortney et al. 2008).

The fraction of short-timescale events due to freefloating planets is the most robust statistic we can infer from the available data (see Section 5). In order to determine an occurrence rate of free-floating planets, we must necessarily assume something about their mass function (for which there is currently no observational constraints). We choose to adopt a free-floating planet mass function that is a delta function at $2 M_{\mathrm{Jup}}$, as this (roughly) reproduces the residual timescale distribution after subtraction of our canonical LMF and wide-separation, bound planets that are not expected to show evidence of a primary. Under this assumption, we compute the number of free-floating planets per mainsequence star and find a median value $N_{\mathrm{ff}}=1.4(0.48-1.8$ at $95 \%$ confidence) in the "hot-start" case and $N_{\mathrm{ff}}=1.2$ (0.29-1.8 at 95\% confidence) for the "cold-start" case.

These values are slightly lower than that suggested by Sumi et al. (2011) of $1.8_{-0.8}^{+1.7}$, but still seem difficult to explain given our current understanding of formation channels for free-floating planets. Our results also suggest occurrence rates of free-floating planets that is higher by a large factor than that inferred by the SONYC imaging survey of NGC 1333 (Scholz et al. 2012b), but quite a bit lower than that inferred by the photometric survey of the $\rho$ Oph cloud core by Marsh et al. (2010). Potential reasons for the differences in frequencies of free-floating planets between our results and those of imaging surveys are 1) imaging surveys are probing a different pop- 
ulation of free-floating, planetary-mass objects, 2) these frequencies are heavily dependent on the local environmental conditions, 3 ) the imaging surveys, which are only typically sensitive to objects more massive than about a couple Jupiter masses, lack the sensitivity to probe the free-floating planet population inferred by microlensing.

Future observations will be critical to further elucidate the true abundance and demographics of free-floating planets. Direct mass measurements of a statisticallysignificant sample of short-timescale microlensing events will allow us to infer the mass function of free-floating planets. It is unlikely that there will be any lens mass measurements from short-timescale microlensing events from the K2 Campaign 9 dataset (see Section 5), but as Penny et al. (2016) point out, K2C9 can still test the hypothesis that these events are, in fact, due to planetary-mass objects, and if so, whether or not they are bound to stars. Ultimately the microlensing survey of WFIRST will provide robust measurements of the freefloating planet mass function, their occurrence rates, and their Galactic distribution (Spergel et al. 2015).

We thank Takahiro Sumi and Radek Poleski for helpful conversations. This research has made use of NASA's Astrophysics Data System and was partially supported by NSF CAREER Grant AST-1056524. Work by CDC was supported in part by an appointment to the NASA Postdoctoral Program at Ames Research Center that is administered by the Universities Space Research Association through a contract with NASA.

\section{REFERENCES}

Adams, F. C., \& Fatuzzo, M. 1996, ApJ, 464, 256

Allers, K. N., Jaffe, D. T., Luhman, K. L., et al. 2007, ApJ, 657, 511

Antognini, J. M. O., \& Thompson, T. A. 2016, MNRAS, 456, 4219

Baraffe, I., Chabrier, G., Barman, T. S., Allard, F., \& Hauschildt, P. H. 2003, A\&A, 402, 701

Bastian, N., Covey, K. R., \& Meyer, M. R. 2010, ARA\&A, 48, 339

Bate, M. R., \& Bonnell, I. A. 2005, MNRAS, 356, 1201

Bennett, D. P., Sumi, T., Bond, I. A., et al. 2012, ApJ, 757, 119

Bihain, G., Rebolo, R., Zapatero Osorio, M. R., et al. 2009, A\&A, 506, 1169

Biller, B. A., Liu, M. C., Wahhaj, Z., et al. 2013, ApJ, 777, 160

Bond, I. A., Abe, F., Dodd, R. J., et al. 2001, MNRAS, 327, 868

Bonfils, X., Delfosse, X., Udry, S., et al. 2013, A\&A, 549, A109

Bowler, B. P., Liu, M. C., Shkolnik, E. L., \& Tamura, M. 2015, ApJS, 216, 7

Burgess, A. S. M., Moraux, E., Bouvier, J., et al. 2009, A\&A, 508,823

Cassan, A., Kubas, D., Beaulieu, J.-P., et al. 2012, Nature, 481, 167

Clanton, C., \& Gaudi, B. S. 2014a, ApJ, 791, 90

Clanton, C., \& Gaudi, B. S. 2014b, ApJ, 791, 91

Clanton, C., \& Gaudi, B. S. 2016, ApJ, 819, 125

Comeron, F., Rieke, G. H., Burrows, A., \& Rieke, M. J. 1993, ApJ, 416, 185

Ford, E. B., Rasio, F. A., \& Yu, K. 2003, Scientific Frontiers in Research on Extrasolar Planets, 294, 181

Fortney, J. J., Marley, M. S., Saumon, D., \& Lodders, K. 2008, ApJ, 683, 1104-1116

Gardner, J. P., Mather, J. C., Clampin, M., et al. 2006, Space Sci. Rev., 123, 485

Gaudi, B. S. 2012, ARA\&A, 50, 411

Gaudi, B. S., Bennett, D. P., Udalski, A., et al. 2008, Science, 319,927

Geers, V., Scholz, A., Jayawardhana, R., et al. 2011, ApJ, 726, 23
Gould, A. 2000, ApJ, 535, 928

Gould, A., Dong, S., Gaudi, B. S., et al. 2010, ApJ, 720, 1073

Gould, A., Gaudi, B. S., \& Han, C. 2003, ApJ, 591, L53

Han, C. 2006, ApJ, 638, 1080

Han, C., Gaudi, B. S., An, J. H., \& Gould, A. 2005, ApJ, 618, 962

Han, C., \& Gould, A. 1995a, ApJ, 449, 521

Han, C., \& Gould, A. 1995b, ApJ, 447, 53

Han, C., \& Gould, A. 2003, ApJ, 592, 172

Han, C., \& Kang, Y. W. 2003, ApJ, 596, 1320

Han, C., Udalski, A., Choi, J.-Y., et al. 2013, ApJ, 762, L28

Henderson, C. B., \& Shvartzvald, Y. 2016, arXiv:1603.05249

Henderson, C. B., Poleski, R., Penny, M., et al. 2015, arXiv:1512.09142

Hillenbrand, L. A., \& Carpenter, J. M. 2000, ApJ, 540, 236

Horner, S. D., \& Rieke, M. J. 2004, Proc. SPIE, 5487, 628

Itoh, Y., Tamura, M., \& Gatley, I. 1996, ApJ, 465, L129

Kirkpatrick, J. D., Barman, T. S., Burgasser, A. J., et al. 2006, ApJ, 639, 1120

Krist, J. E., Beichman, C. A., Trauger, J. T., et al. 2007, Proc. SPIE, 6693, 66930H

Lafrenière, D., Doyon, R., Marois, C., et al. 2007, ApJ, 670, 1367

Laughlin, G., Bodenheimer, P., \& Adams, F. C. 2004, ApJ, 612, L73

Lucas, P. W., \& Roche, P. F. 2000, MNRAS, 314, 858

Luhman, K. L., Wilson, J. C., Brandner, W., et al. 2006, ApJ, 649,894

Luhman, K. L., Allers, K. N., Jaffe, D. T., et al. 2007a, ApJ, 659, 1629

Luhman, K. L., Joergens, V., Lada, C., et al. 2007b, Protostars and Planets V, 443

Marsh, K. A., Plavchan, P., Kirkpatrick, J. D., et al. 2010, ApJ, 719,550

McGovern, M. R., Kirkpatrick, J. D., McLean, I. S., et al. 2004, ApJ, 600, 1020

Montet, B. T., Crepp, J. R., Johnson, J. A., Howard, A. W., \& Marcy, G. W. 2014, ApJ, 781, 28

Mustill, A. J., Veras, D., \& Villaver, E. 2014, MNRAS, 437, 1404

Mužić, K., Scholz, A., Geers, V., Fissel, L., \& Jayawardhana, R. 2011, ApJ, 732, 86

Mužić, K., Scholz, A., Geers, V. C., Jayawardhana, R., \& López Martí, B. 2014, ApJ, 785, 159

Mužić, K., Scholz, A., Geers, V., Jayawardhana, R., \& Tamura, M. 2012, ApJ, 744, 134

Mužić, K., Scholz, A., Geers, V. C., \& Jayawardhana, R. 2015, ApJ, 810, 159

Nordh, L., Olofsson, G., Abergel, A., et al. 1996, A\&A, 315, L185

Öpik, E. 1924, Publications de l'Observatoire Astronomique de l'Universitê de Tartr, 25, No. 6, 1

Padoan, P., Nordlund, A., \& Jones, B. J. T. 1997, MNRAS, 288, 145

Penny, M. T., Rattenbury, N. J., Gaudi, B. S., \& Kerins, E. 2016, arXiv:1605.01059

Quanz, S. P., Goldman, B., Henning, T., et al. 2010, ApJ, 708, 770

Quanz, S. P., Lafrenière, D., Meyer, M. R., Reggiani, M. M., \& Buenzli, E. 2012, A\&A, 541, A133

Raymond, S. N., Barnes, R., Armitage, P. J., \& Gorelick, N. 2008, ApJ, 687, L107

Sako, T., Sekiguchi, T., Sasaki, M., et al. 2008, Experimental Astronomy, 22, 51

Schlieder, J. E., Beichman, C. A., Meyer, M. R., \& Greene, T. 2016, IAU Symposium, 314, 288

Scholz, A., Geers, V., Jayawardhana, R., et al. 2009, ApJ, 702 , 805

Scholz, A., Jayawardhana, R., Muzic, K., et al. 2012a, ApJ, 756, 24

Scholz, A., Muzic, K., Geers, V., et al. 2012b, ApJ, 744, 6

Silk, J. 1977, ApJ, 214, 718

Spergel, D., Gehrels, N., Baltay, C., et al. 2015, arXiv:1503.03757

Sumi, T., Abe, F., Bond, I. A., et al. 2003, ApJ, 591, 204

Sumi, T., Bennett, D. P., Bond, I. A., et al. 2010, ApJ, 710, 1641

Sumi, T., Bennett, D. P., Bond, I. A., et al. 2013, ApJ, 778, 150

Sumi, T., Kamiya, K., Bennett, D. P., et al. 2011, Nature, 473, 349

Tamura, M., Itoh, Y., Oasa, Y., \& Nakajima, T. 1998, Science, 282,1095 
Udalski, A. 2003, AcA, 53, 291

Veras, D., \& Raymond, S. N. 2012, MNRAS, 421, L117

Veras, D., Wyatt, M. C., Mustill, A. J., Bonsor, A., \& Eldridge, J. J. 2011, MNRAS, 417, 2104

Weights, D. J., Lucas, P. W., Roche, P. F., Pinfield, D. J., \& Riddick, F. 2009, MNRAS, 392, 817

Wyrzykowski, Ł., Rynkiewicz, A. E., Skowron, J., et al. 2015, ApJS, 216, 12
Yee, J. C. 2013, ApJ, 770, L31

Zapatero Osorio, M. R., Béjar, V. J. S., Martín, E. L., et al. 2000, Science, 290, 103

Zapatero Osorio, M. R., Béjar, V. J. S., Martín, E. L., et al. 2002, ApJ, 578, 536 